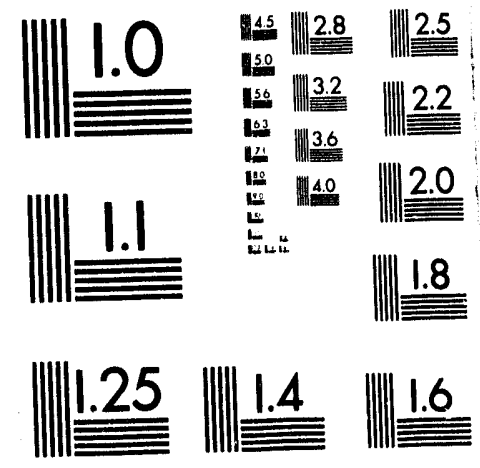



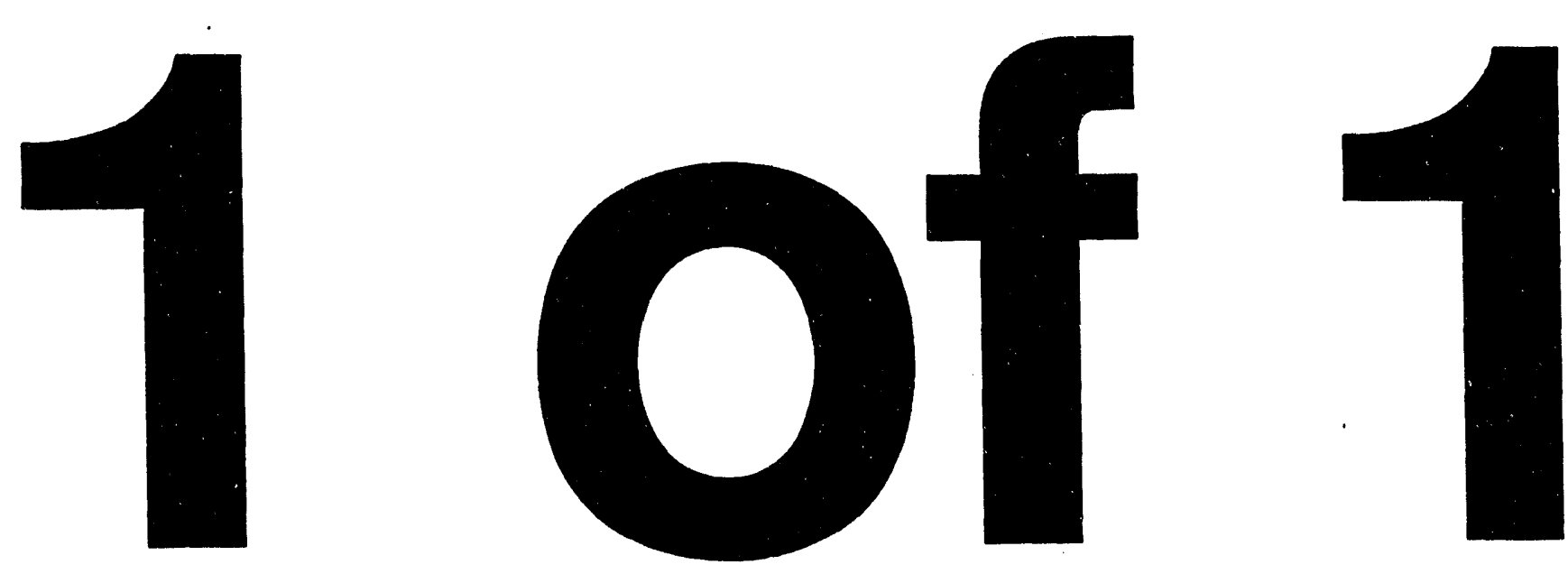
UC-414 and UC-406

Issued: April 1994

Simulation of the GEM Central Tracker

Performance Using GEANT

M. L. Brooks 


\title{
SIMULATION OF THE GEM CENTRAL TRACKER PERFORMANCE USING GEANT
}

\author{
by
}

\author{
M. L. Brooks
}

\begin{abstract}
The performance of the GEM central tracker in terms of momentum, impact parameter, and $\mathrm{z}$ vertex resolution has been determined using a GEANT based simulation program. The resolutions will be presented for both the full tracker which includes a silicon portion and an IPC portion and for the $10^{34}$ configuration which is comprised of only IPC chambers. The resolution of electrons vs. muons will also be presented.
\end{abstract}

\section{INTRODUCTION}

The GEM (Gamma Electron Muon) central tracker (one of the proposed detectors for the Superconducting Supercollider (SSC)) has been simulated using the high energy physics Monte Carlo code GEANT. This simulation has been used to determine the performance of the central tracker in terms of momentum resolution, impact parameter, and $\mathrm{z}$ vertex resolution over the entire eta range of the detector for both electrons and muons of momentum $(1,500) \mathrm{GeV} / \mathrm{c}$. The resolutions have also been determined separately for the $10^{33} \mathrm{~cm}^{-2} \mathrm{~s}^{-1}$ luminosity configuration which includes a silicon tracker as well as an outer interpolating pad chamber (IPC) tracker, and for the $10^{34} \mathrm{~cm}^{-2} \mathrm{~s}^{-1}$ configuration which consists of just an IPC tracker. A brief overview of the code, which has been described in detail previously ("Simulation of the GEM Silicon Central Tracker," LA-12714-MS), will be given and the results will be presented.

\section{SIMULATION OF THE TRACKER}

The GEM central tracker consists of an inner tracker which is made of silicon strip wafers and an outer tracker of interpolating pad chambers (IPCs). In the full central tracker (which will all be operational at $10^{33} \mathrm{~cm}^{-2} \mathrm{~s}^{-1}$ ) the central silicon region contains six layers of stereo pairs of silicon extending $\pm 18 \mathrm{~cm}$ in $\mathrm{z}$ and four layers of IPC stereo pairs which extend to $\pm 100 \mathrm{~cm}$ in $\mathrm{z}$. The silicon barrel layers are located at radii of 10 , $12,15,17,20$, and $22 \mathrm{~cm}$. The pad layers are located at radii of approximately $45,57,65$ and $78 \mathrm{~cm}$. Each stereo pair in both the silicon and the pad regions produces one three dimensional coordinate for the pattern recognition. The forward silicon disks cover $\mathrm{z}= \pm 20 \mathrm{~cm}$ to $\mathrm{z}= \pm 87 \mathrm{~cm}$ and the forward IPC disks cover $\mathrm{z}= \pm 113 \mathrm{~cm}$ to $\mathrm{z}= \pm 168 \mathrm{~cm}$. The forward silicon disks extend from $10 \mathrm{~cm}$ to $26 \mathrm{~cm}$ radially except for the most forward disks which extend from $10 \mathrm{~cm}$ to $24 \mathrm{~cm}$. The forward pad chambers extend from approximately $20 \mathrm{~cm}$ to $79 \mathrm{~cm}$ radially. Great care has been taken in the GEANT code to accurately represent both the detectors themselves as well as all nonsensitive 
materials which are expected to be present in the final tracker. These nonsensitive volumes include
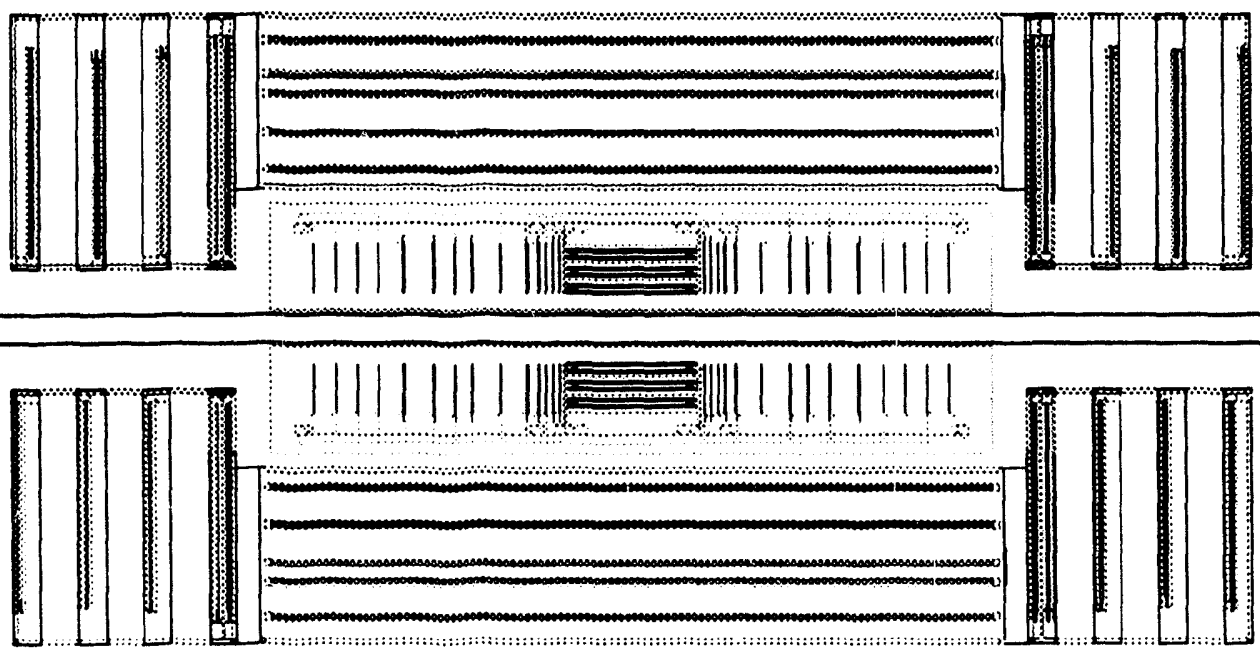

Figure 1. The geometry of the GEM central tracker as produced by GEANT.

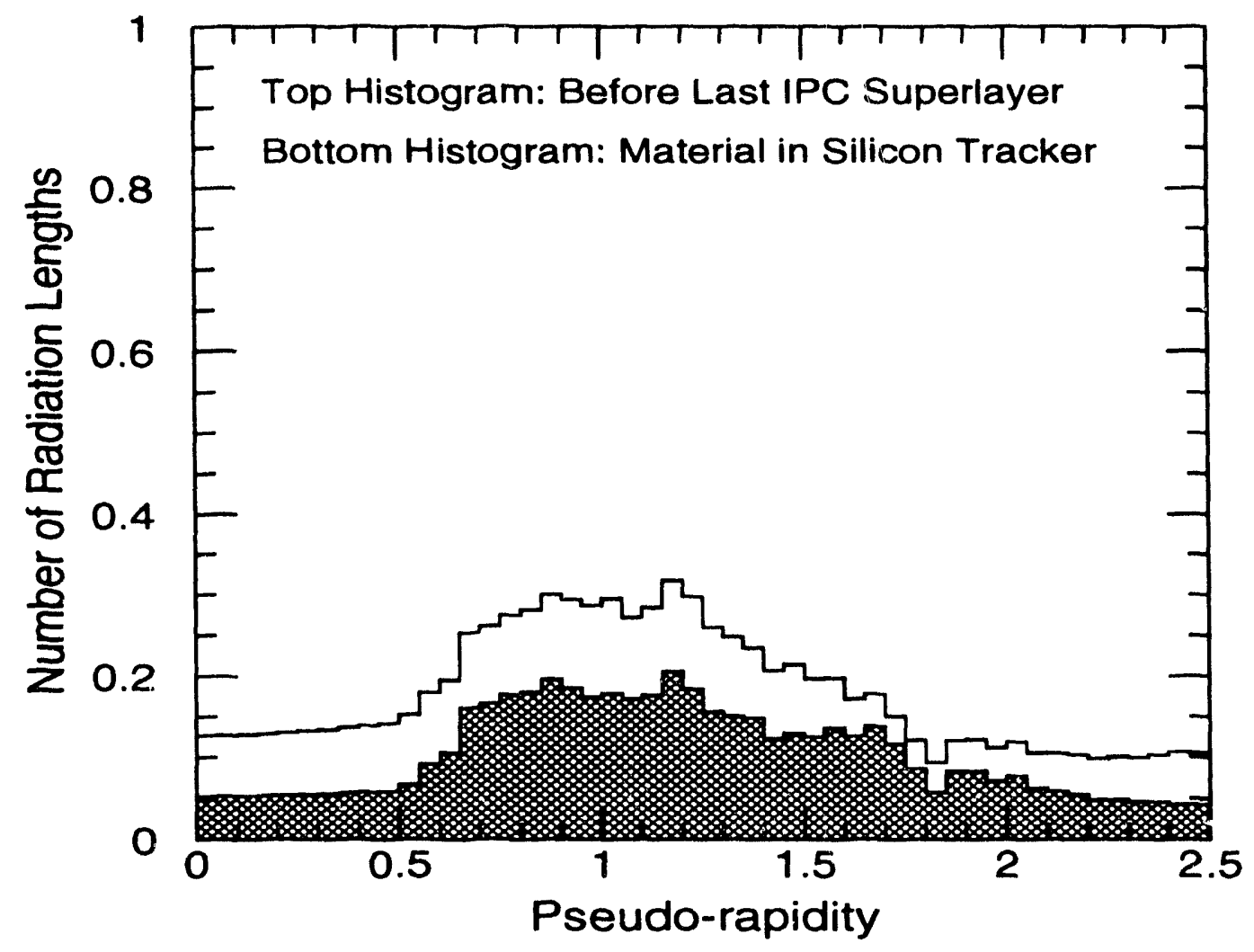

Figure 2. Material, in units of radiation length, in the central tracker. The bottom curve is the silicon detector only; the upper curve includes the IPC tracker up to the outermost layer. 
the electronics for the detectors, the cooling materials, all cabling, the support structures, the gas enclosure for the silicon portion of the tracker, and the beam pipe. A picture of the geometry, produced by GEANT, can be seen in Figure 1.

The central tracker has been designed so that a minimum amount of material is used, while still maintaining the mechanical stability and electrical requirements. The resulting material contributions to the GEM tracker (as produced by GEANT) are shown in Figure 2 where the radiation length of the tracker vs. eta is shown for the silicon portion and for the full tracker which includes the IPCs.

\section{DETECTOR RESOLUTIONS}

\subsection{Momentum Resolution}

The momentum resolution of the tracker has been determined by using pattern recognition to find tracks (as described in LA-12678-MS, "Pattern Recognition in the GEM Central Tracker at Luminosity of $10^{33} \mathrm{~cm}^{-2} \mathrm{~s}^{-11}$ ), fitting the coordinate points of the track to a helix and extracting the momentum given the radius of curvature of the helix and the magnetic field of our tracker. The error in the momentum calculation was calculated from

$$
\frac{\Delta(1 / \mathrm{p})}{(1 / \mathrm{p})}=\mathrm{pT}_{\mathrm{T}, \text { true }} *\left[\frac{\mathrm{Q}_{\text {meas }}{ }^{*} \mathrm{Q}_{\text {true }}}{\mathrm{pT}}-\frac{1}{\mathrm{p}, \mathrm{Ttrue}}\right]
$$

where $\mathrm{p}_{\text {T, true }}$ is the true momentum in the transverse direction, $\mathrm{Qmeas}_{\text {is }}$ is the meared sign of the charge of the track, Qtrue is the true sign, and pT is the measured transverse momentum. This error is comparable to the error in the sagitta measurement and should be Gaussian for all momenta. The momentum resolutions that will be presented here are the sigma's of the distributions of this calculated $\Delta(1 / p) /(1 / p)$ for various momentum and eta intervals.

The momentum resolution of the GEM tracker has been calculated separately for muons and electrons since the bremsstrahlung of the electrons causes the electrons to lose more energy as they pass through the tracker than the muons do, thus causing a degradation in momentum resolution of the electrons relative to the muons. The muon momentum resolution is limited at low momentum by multiple scattering in the tracker volume and at high momentum by the intrinsic resolutions of the detectors, the magnetic field in the tracker, and the radial extent of the tracker layers. The momentum resolution multiple scattering limit of the tracker has been determined to be $3.5 \%$ and the resolution at high momenta is $0.0015 \mathrm{p}$ :

$$
\frac{\sigma}{p}=\sqrt{(0.00115 p)^{2}+(0.035)^{2}}
$$

The resolution at $\eta=0$ can be seen in Figure 3 where the momentum resolution is shown as a function of momentum along with the parameterized fit that was given above. The resolution has also been calculated as a function of eta, and this can be seen in Figure 4 where the resolution is shown for $p_{\text {total }}=20 \mathrm{GeV} / \mathrm{c}$ and $\mathrm{p}_{\mathrm{T}}=20 \mathrm{GeV} / \mathrm{c}$. The resolution is flat vs. eta until about $\eta=1.5$. At this point, the length of the track in the transverse 
plane is truncated by the end of the tracker, and the resolution degrades because of the decreased value of $B \times L$.

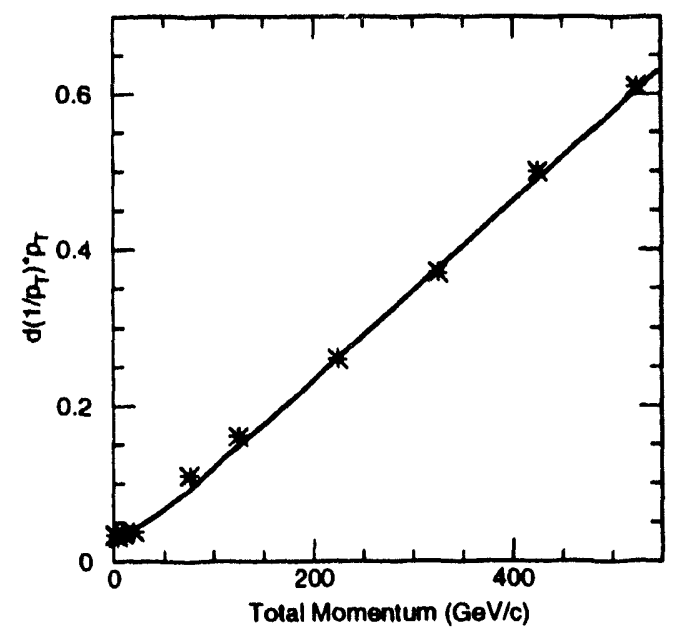

Figure 3. The momentum resolution of the central tracker vs. momentum at $\eta=0$.

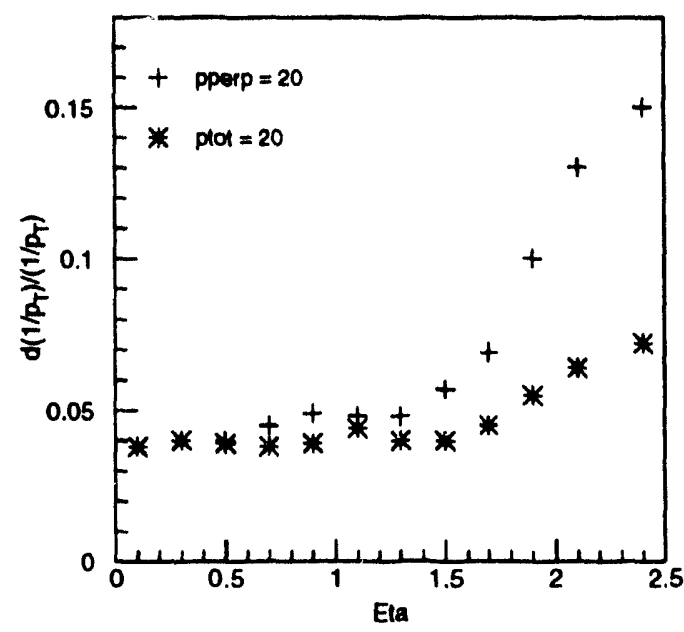

Figure 4. The momentum resolution of the central tracker vs. $\eta$ for $p$ tot $=20$ and $\mathrm{pT}=20 \mathrm{GeV} / \mathrm{c}$.

The momentum resolution distributions for electrons have also been calculated for momentum $=(1,500) \mathrm{GeV} / \mathrm{c}$ and eta $=(0,2.5)$. The shape of the distributions has been parameterized and is used in a "fast" Monte Carlo for physics studies. A comparison of the resolution distributions for electrons and muons at $\eta=0$ for momentum $=1,10$, and $100 \mathrm{GeV} / \mathrm{c}$ can be seen in Figure 5. As can be seen, the central portions of the distributions are very similar, but there is a tail of poorly measured tracks in the electron spectra that come from electrons which have suffered bremsstrahlung in the volume of the tracker.

The momentum calculations have been used to determine the ability of the tracker to determine the sign of the charge of particles. Figure 6 shows the total momentum and transverse momentum values at which $95 \%$ of the tracks in the tracker have the sign correctly determined for electrons and muons. As can be seen, the charge sign determination is actually slightly better for electrons than for muons. This is because the energy loss to bremsstrahlung of the electrons causes the track on average to curve slightly more in the magnetic field than a muon of the same momentum would, allowing the charge sign of the electron to be determined correctly slightly more often than for muons.

The momentum resolution for muons in the $10^{34}$ tracker configuration (only IPCs) vs. momentum is shown in Figure 7 along with the momentum resolution for the $10^{33}$ configuration. The resolution can be parameterized by

$$
\frac{\sigma}{p}=\sqrt{(0.0025 p)^{2}+(0.025)^{2}}
$$


Muons
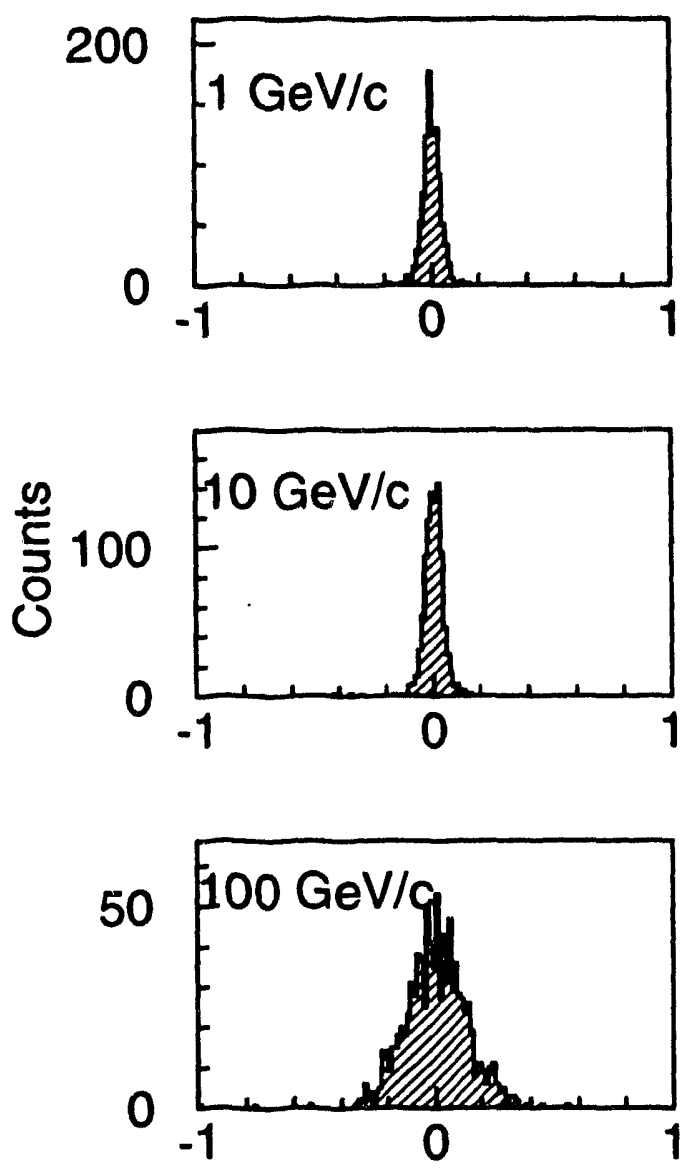

Electrons
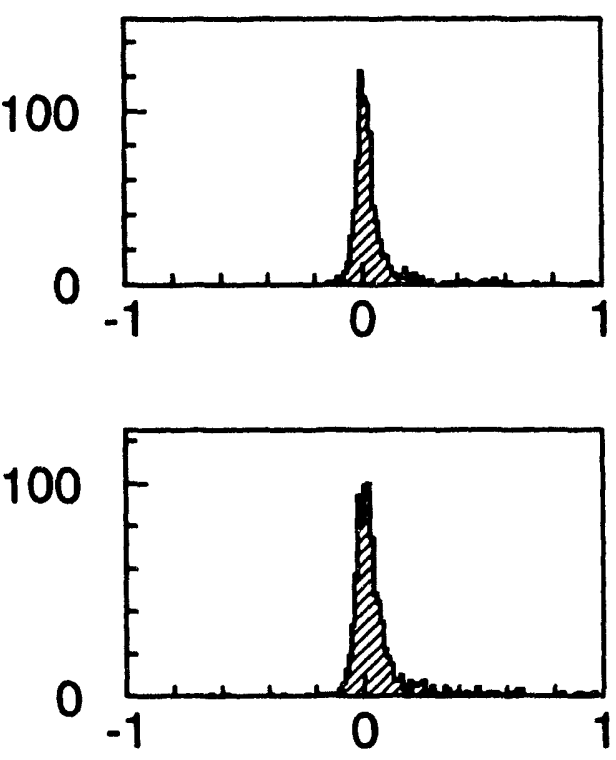

50

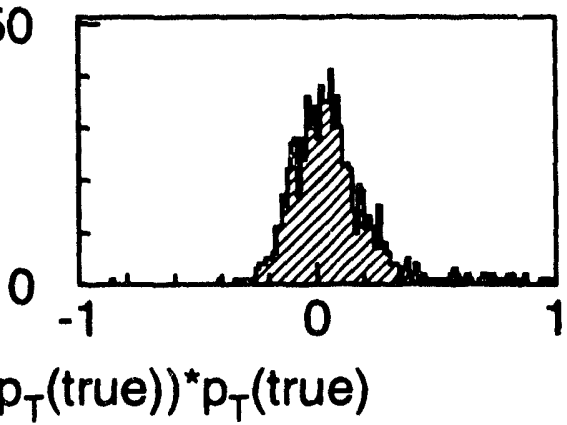

Figure 5. The momentum resolution distribution for electrons and muons of momentum $=1,10$, and $100 \mathrm{GeV} / \mathrm{c}$ at $\eta=0$.

and is roughly twice the resolution that is obtained for the full tracker at high momenta, but has a slightly better multiple scattering limit because it was assumed that the silicon portion of the tracker would be removed from the tracker volume at $10^{34}$. The momentum resolution vs. eta is shown in Figure 8.

\subsection{Impact Parameter Resolution}

The impact parameter, which is defined as the distance of closest approach in the $x-y$ plane of the measured track to the true vertex of the track, has been calculated over the eta range of the tracker and for momentum $=(1,500) \mathrm{GeV} / \mathrm{c}$. The resulting resolution at $\eta=0$, as a function of momentum can be seen in Figure 9. The impact parameter resolution vs. momentum can be parameterized by:

$$
\sigma_{b}=24 \sqrt{1+\frac{70}{p^{2}}} \mu m
$$




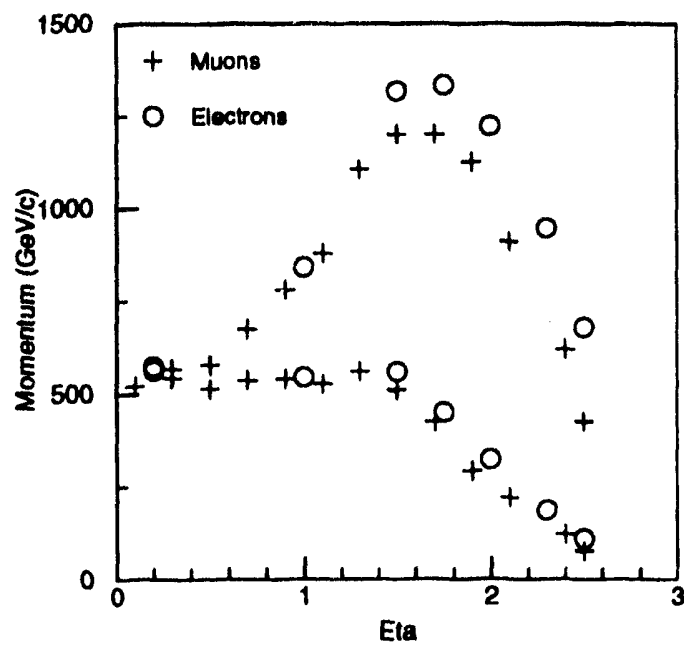

Figure 6. The total momentum (upper curves) and transverse momentum (lower curves) values at which $95 \%$ of the tracks in the central tracker have their charge sign determined correctly for electrons and muons vs. $\eta$.

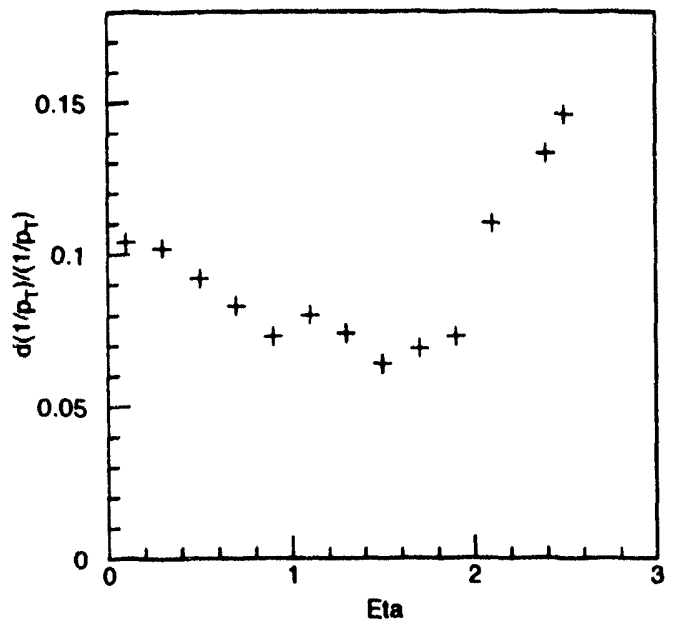

Figure 8. The momentem resolution of the central tracker in the $10^{34}$ configuration vs. eta for $p_{\text {tot }}=20 \mathrm{GeV} / \mathrm{c}$.

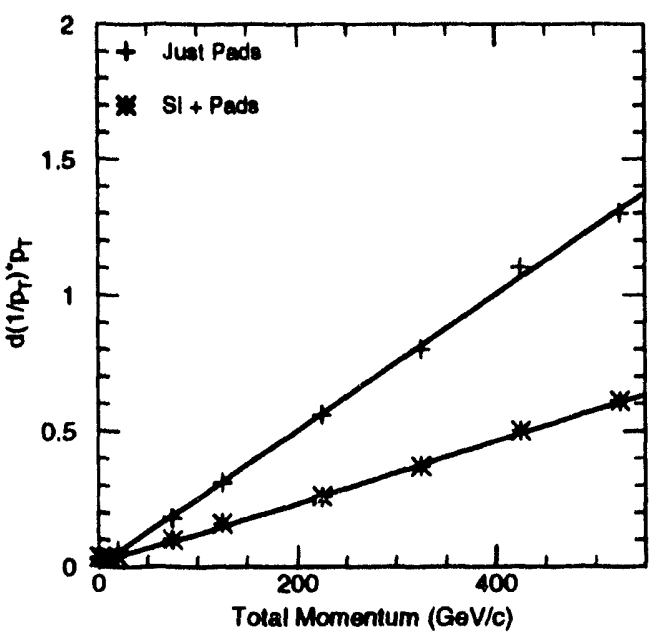

Figure 7. The momentum resolution of the central tracker in the $10^{34}$ configuration vs. momentum, at $\eta=0$. The resolution for the full tracker is shown for comparison.

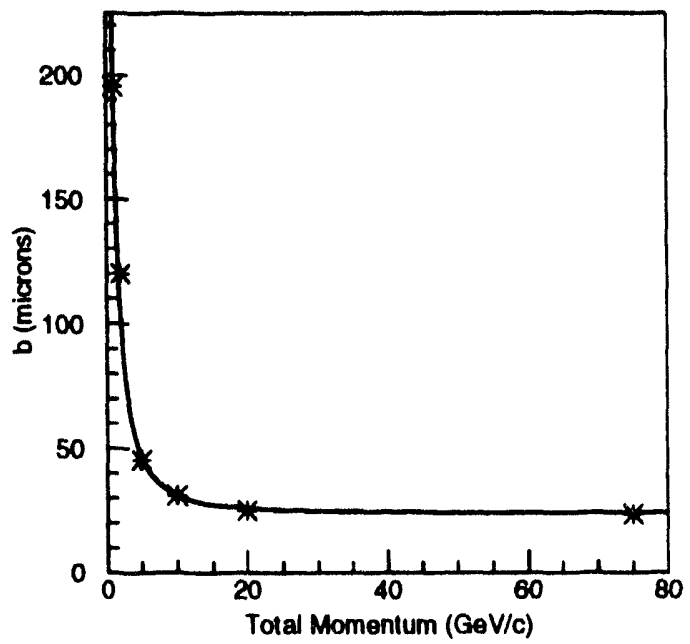

Figure 9. The impact parameter resolution of the full central tracker vs. momentum at $\eta=0$. 


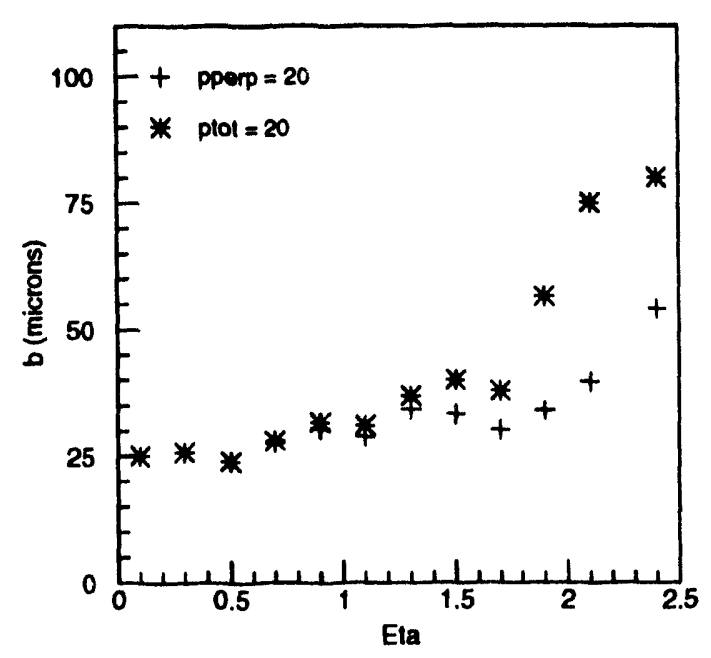

Figure 10. The impact parameter resolution for the full central tracker vs. $\eta$ for $p_{\text {tot }}=20 \mathrm{GeV} / \mathrm{c}$ and $\mathrm{pT}=20 \mathrm{GeV} / \mathrm{c}$.

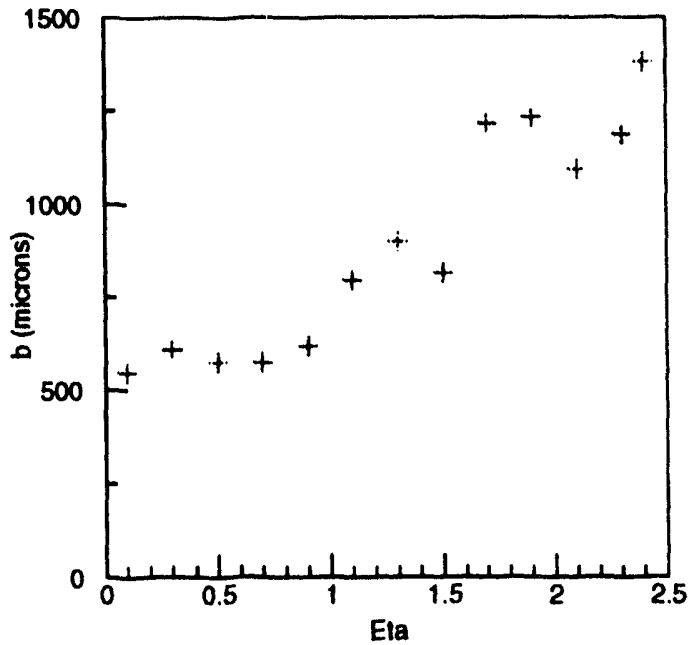

Figure 12. The impact parameter resolution of the central tracker in the $10^{34}$ configuration vs. $\eta$ for $p_{\text {tot }}=20 \mathrm{GeV} / \mathrm{c}$.

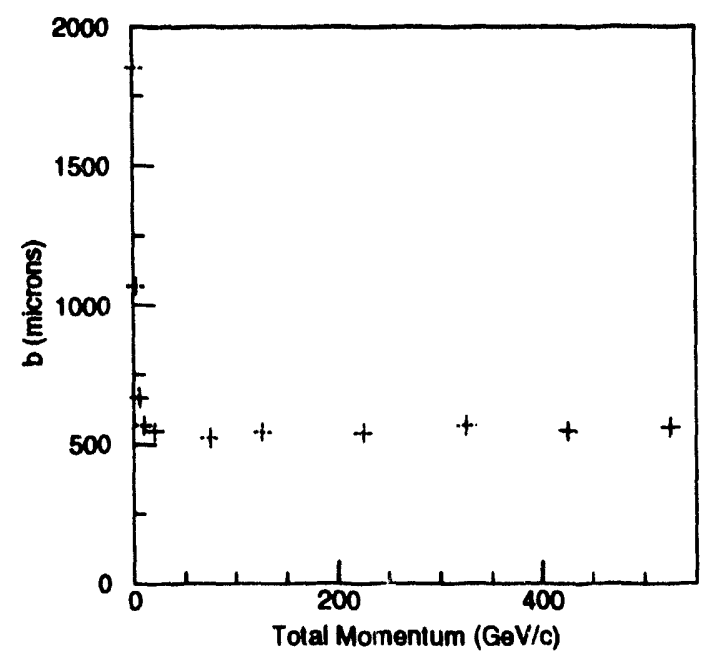

Figure 11. The impact parameter resolution of the tracker in the $10^{34}$ configuration vs. momentum at $\eta=0$.

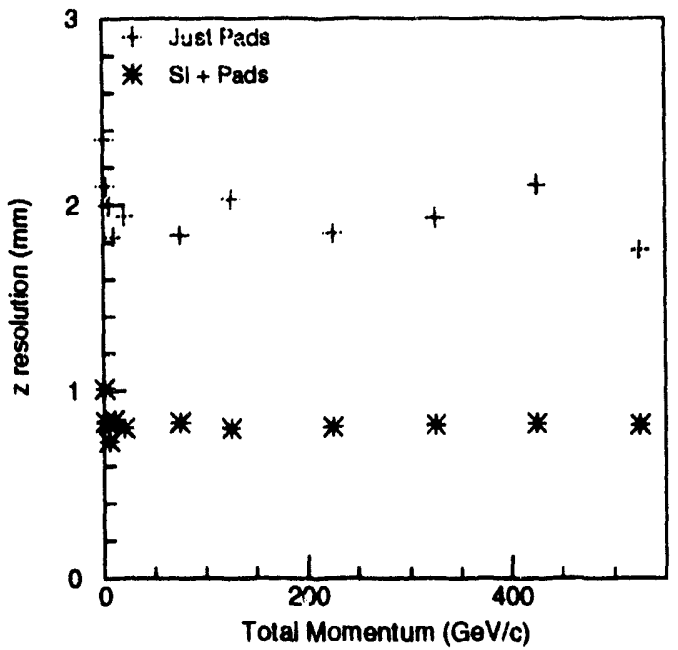

Figure 13. The $\mathrm{z}$ resolution of the full central tracker and the tracker in the $10^{34}$ configuration vs. momentum at $\eta=0$.

where $\mathrm{p}$ is the total momentum of the track in $\mathrm{GeV} / \mathrm{c}$. The resolution is approximately $25 \mu \mathrm{m}$ for momenta above $20 \mathrm{GeV} / \mathrm{c}$ and degrades at low momenta because of multiple scattering in the detector. The impact parameter resolution vs. eta, for $p_{\text {total }}=20 \mathrm{GeV} / \mathrm{c}$ and $\mathrm{pT}_{\mathrm{T}}=20 \mathrm{GeV} / \mathrm{c}$ is shown in Figure 10. Again, the resolution is flat from $\eta=0$ to $\eta=1.5$ and then the resolution degrades because the curvature of the track is more poorly measured at large eta. 
The impact parameter resolution vs. momentum and eta for the $10^{34}$ tracker configuration is shown in Figures 11 and 12.

\subsection{Z Vertex Resolution}

The resolution in the $z$ vertex has a so been calculated for the tracker. The $z$ vertex resolution is the difference between the measured $\mathrm{z}$ value of the track at the distance of closest approach in the $x-y$ plane to the true vertex and the $z$ value of the true vertex. The $z$ resolution at $\eta=0$ vs. momentum for both the $10^{33}$ tracker and the $10^{34}$ tracker is shown in Figure 13. We obtain a resolution of approximately $0.8 \mathrm{~mm}$ at $10^{33}$ and $2 \mathrm{~mm}$ at $10^{34}$. The resolution vs. eta for each tracker configuration is shown in Figure 14. Again, the resolution is flat from $\eta=(0,1.5)$ and degrades at larger eta. The

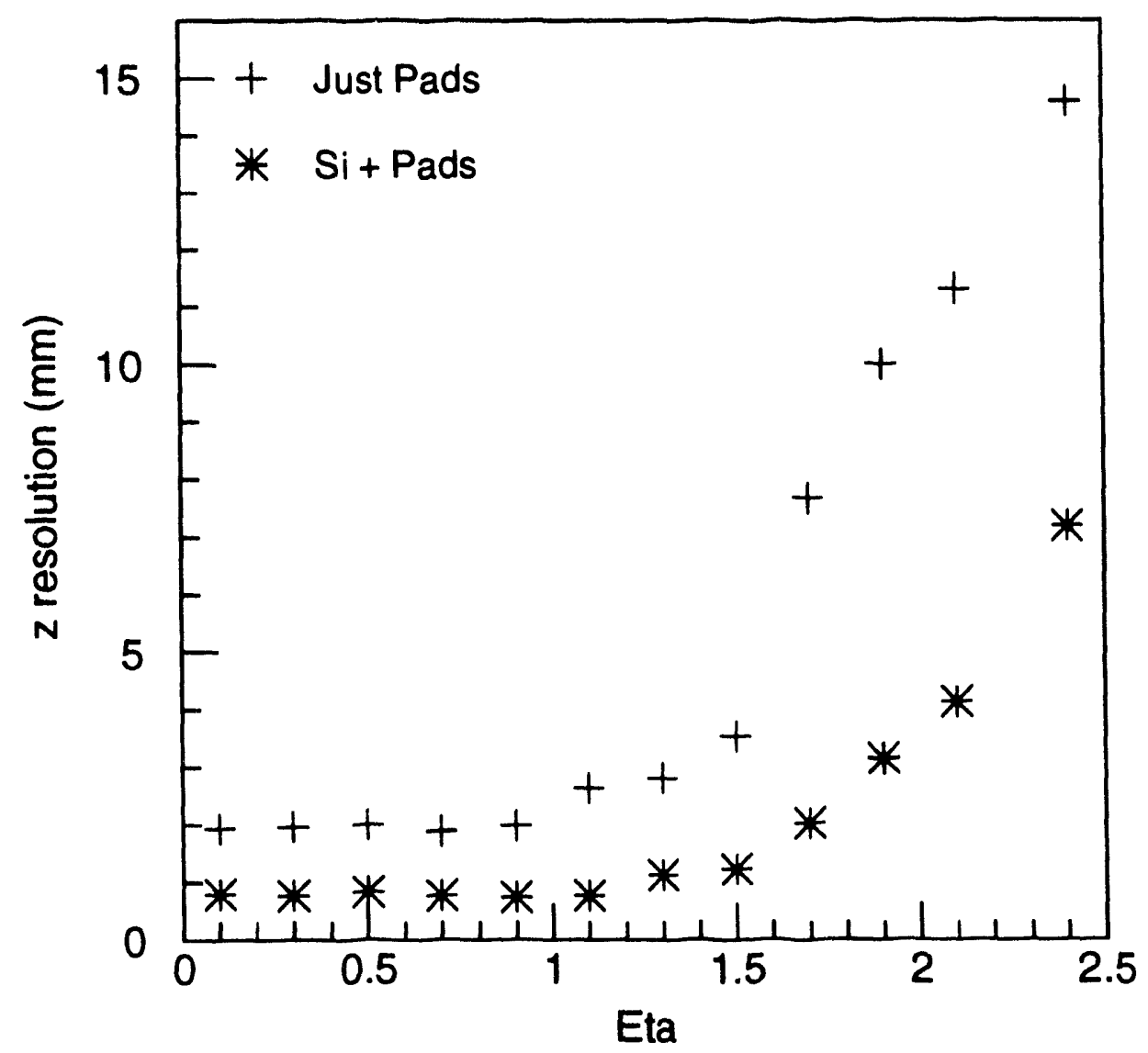

Figure 14. The $\mathrm{z}$ resolution of the full central tracker and the tracker in the $10^{34}$ configuration vs. $\eta$ for $p_{t a t}=20 \mathrm{GeV} / \mathrm{c}$.

degradation is more severe for the $\mathrm{z}$ vertex resolution than for the momentum and impact parameter resolutions because not only are the track parameters measured more poorly at large eta because of the truncation of the track in the transverse plane, but also the error in the projection to the $\mathrm{z}$ vertex increases as the angle of the track relative to the $\mathrm{z}$ axis 
decreases. This causes the $\mathrm{z}$ resolution to degrade by about a factor of eight from $\eta=0$ to $\eta=2.4$.

\section{CONCLUSION}

The GEM central tracker, with the design presented in this paper, will achieve momentum resolution measurements of $\left[(0.0012 p)^{2}+(0.035)^{2}\right]^{1 / 2}$ at luminosity $=10^{33} \mathrm{~cm}^{-2} \mathrm{~s}^{-1}$ (full tracker), and $\left[(0.0025 \mathrm{p})^{2}+(0.025)^{2}\right]^{1 / 2}$ at luminosity $=10^{34} \mathrm{~cm}^{-2} \mathrm{~s}^{-1}$ (tracker comprised of just pad chambers). The momentum resolution for the full tracker translates into a charge separation capability of $95 \%$ of the tracks below approximately $600 \mathrm{GeV} / \mathrm{c}$ having their sign measured correctly below $\eta=1.5$. The impact parameter resolution achieved at luminosity $=10^{33} \mathrm{~cm}^{-2} \mathrm{~s}^{-1}$ is approximately $24\left[1+(70 / p)^{2}\right]^{1 / 2} \mu \mathrm{m}$ at $\eta=0$, and degrades beyond $\eta=1.5$ because the radial extent of the measurement points on a track decreases in the forward regions. At high luminosity, the impact parameter resolution degrades to a few hundred microns.

The $\mathrm{z}$ resolution of the full tracker is approximately $0.8 \mathrm{~mm}$ below $\eta=1.5$ and degrades at larger eta. The $\mathrm{z}$ resolution for the high luminosity version of the tracker is approximately $2.0 \mathrm{~mm}$ below $\eta=1.5$. 

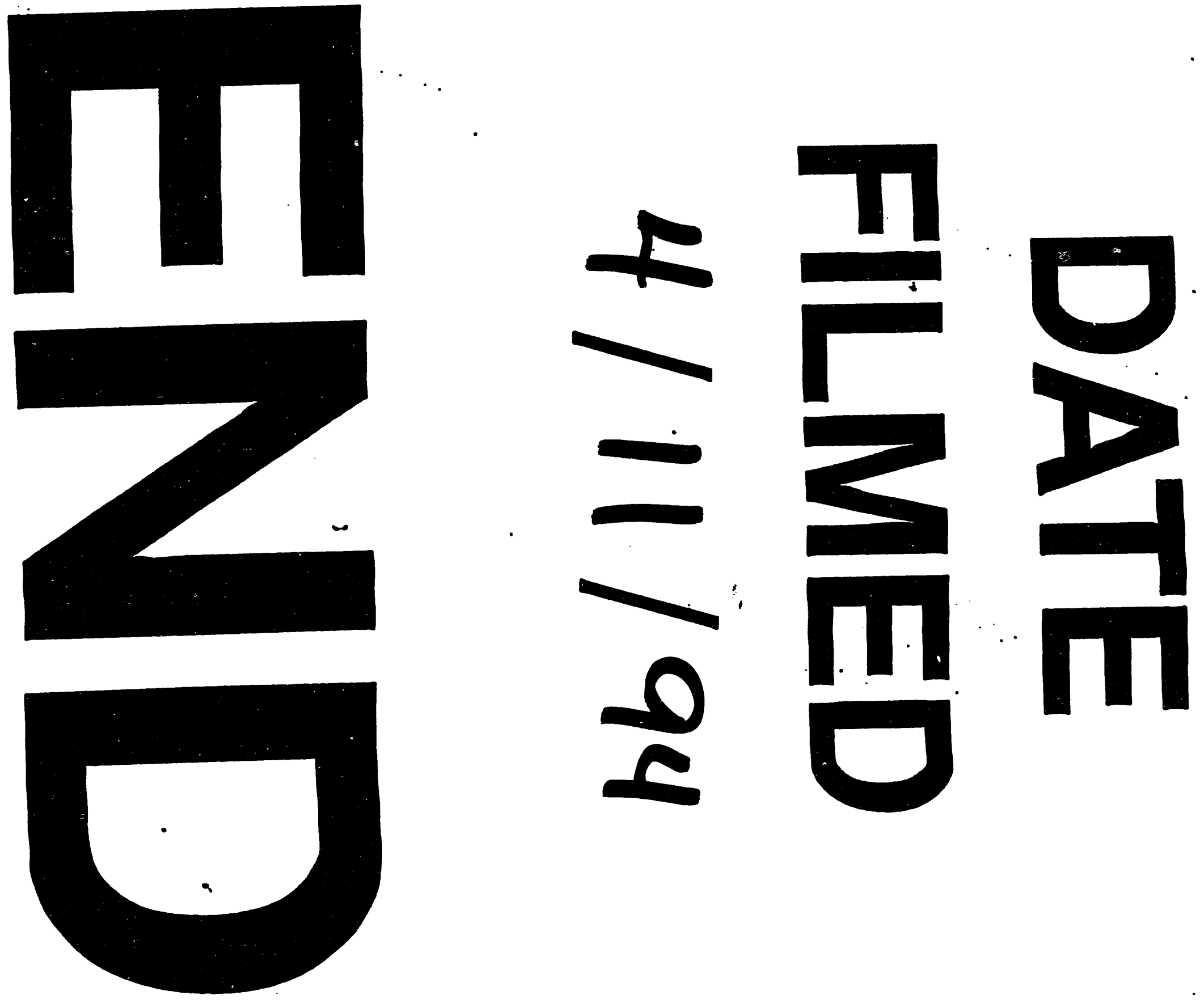